\title{
The Odd Inverse Pareto-Weibull Distribution
}

\author{
Y. AbDelall Yassmen*1 \\ ${ }^{1}$ Department of Mathematical Statistics, Faculty of Graduate Studies for Statistical Research, Cairo \\ University, Egypt
}

\begin{abstract}
The odd inverse Pareto-Weibull distribution is introduced as a new lifetime distribution based on the inverse Pareto and the T-X family. Some mathematical properties of the new distribution are studied. The method of maximum likelihood is used for estimating the model parameters and the observed Fisher's information matrix is derived. The importance and flexibility of the proposed model are assessed using a real data.
\end{abstract}

Keywords inverse Pareto distribution; moments; maximum likelihood; odd inverse Pareto; order statistics

\section{Introduction}

In statistical inference, parametric modeling of data is the main source of obtaining a new family of distributions in hope of adding flexibility for existing ones. So many authors introduced different new families which are modification, generalization or extension of old ones. Some of wellknown families are: Weibull-G (Bourguignon et al., 2014), gamma-G (Zografos and Balakrishnan, 2009), beta-G (Eugene et al., 2002), logistic-G (Torabi and Hedesh, 2012), Kumaraswamy-G (Cordeiro et al., 2017), Lomax-G (Cordeiro et al., 2014), odd Lomax-G (Cordeiro et al., 2019) and exponentiated Weibull-H (Cordeiro et al., 2017) families.

Recently, Aldahlan et al. (2019) introduced a new family of continuous distributions called the odd inverse Pareto-G (OIP-G) family which extends the exponentiated-G (Exp-G) family due to Gupta et al. (1998) and the Marshall-Olkin-G (Mo-G) family due to Marshall and Olkin (1997). The new family is constructed based on the inverse Pareto distribution and the TX family (Alzaatreh et al., 2013). The cumulative distribution function (cdf) and probability density function (pdf) of OIP-G family are defined by :

$$
F(x ; \alpha, \beta, \varphi)=\frac{G(x ; \varphi)^{\alpha}}{[1-G(x ; \varphi)]^{\alpha}}\left[\beta+\frac{G(x ; \varphi)}{1-G(x ; \varphi)}\right]^{-\alpha}, \quad x>0, \quad \alpha>0, \quad \beta>0,
$$

and

$$
f(x ; \alpha, \beta, \varphi)=\alpha \beta g(x ; \varphi) G(x ; \varphi)^{\alpha-1}[\beta+(1-\beta) G(x ; \varphi)]^{-\alpha-1},
$$

where $\alpha$ and $\beta$ are two additional shape parameters, $G(x ; \phi)$ is the baseline cdf with parameter vector $\phi$ and $g(x ; \phi)=d G(x ; \phi) / d x$.

In this paper, we define a new lifetime distribution called the OIP-W distribution. We hope that it will provide consistently better fits than other generated distributions under the same underlying distribution. This paper is organized as follows.

In Section 2, we study the OIP-W distribution. Statistical properties are calculated in Section 3 and 4. Maximum likelihood estimation of the parameters is determined in Section 5. An application of OIP-W distribution for a real data set is performed in Section 6. Finally, conclusion is appeared in Section 7.

\footnotetext{
*Email: yassmenamalek@gmail.com.
} 


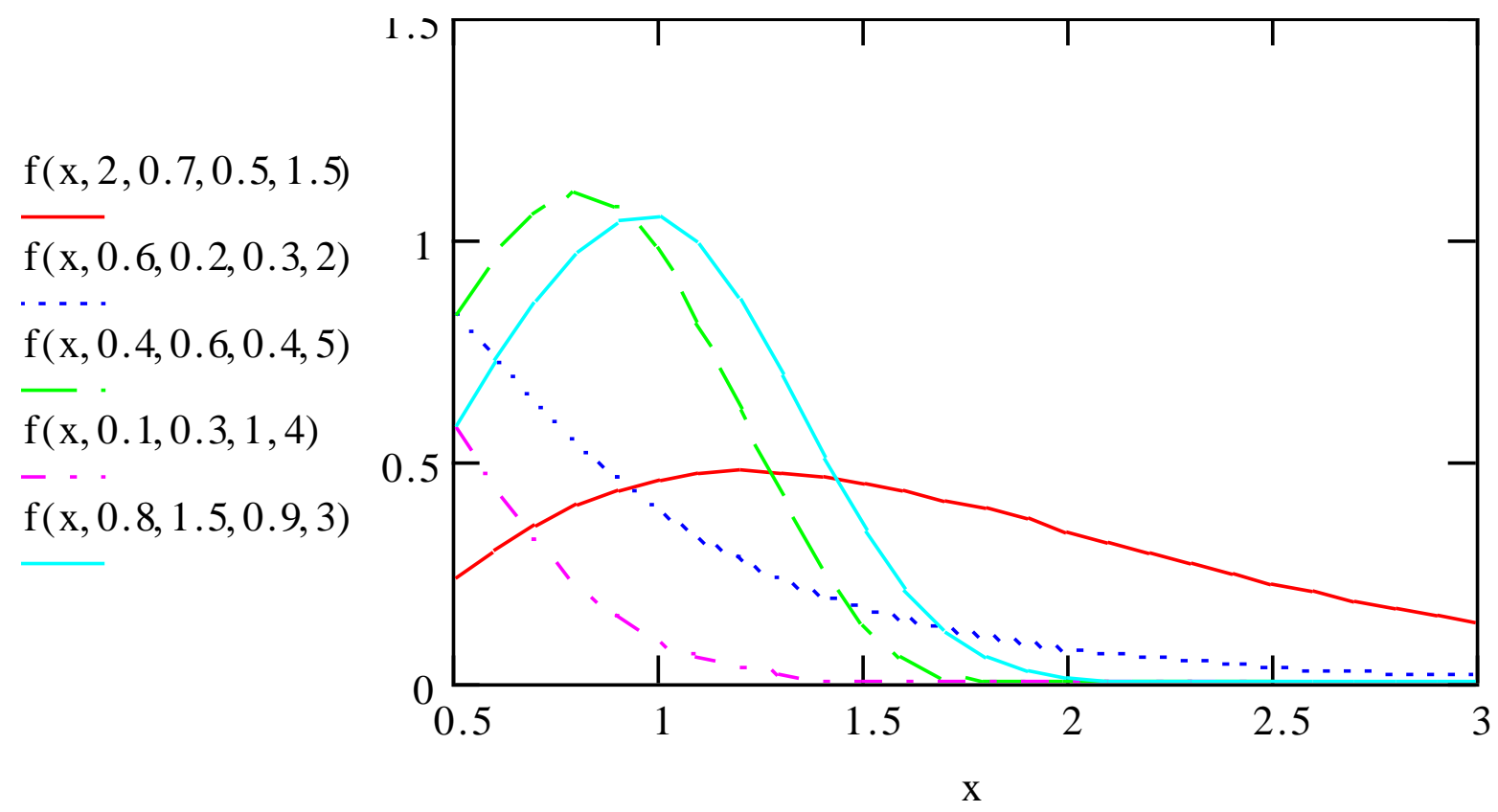

Figure 1: The pdfs of OIP-W distribution with different parameters.

\section{The New Distribution}

The pdf and cdf of weibull (W) distribution with scale parameter $\theta>0$ and shape parameter $\gamma>0$ are given by $g(x ; \theta, \gamma)=\theta \gamma x^{\gamma-1} e^{-\left(\theta x^{\gamma}\right)}$ and $G(x ; \theta, \gamma)=1-e^{-\left(\theta x^{\gamma}\right)}$, respectively. Then, the OIP-W cdf with a set of parameters $\zeta=(\alpha, \beta, \theta, \gamma)$ follows from (1) given by:

$F(x ; \alpha, \beta, \theta, \gamma)=\left[1-e^{-\theta x^{\gamma}}\right]^{\alpha}\left[\beta+(1-\beta)\left(1-e^{-\theta x^{\gamma}}\right)\right]^{-\alpha}, \quad x>0, \alpha>0, \beta>0, \theta>0, \gamma>0$,

The corresponding OIP-G pdf follows from (2) is given by

$$
f(x ; \alpha, \beta, \theta, \gamma)=\alpha \beta \theta \gamma x^{\gamma-1} e^{-\theta x^{\gamma}}\left(1-e^{-\theta x^{\gamma}}\right)^{\alpha-1}\left[\beta+(1-\beta)\left(1-e^{-\theta x^{\gamma}}\right)\right]^{-\alpha-1} .
$$

Also, the survival function and hazard function are given respectively as follows:

$$
S(x ; \alpha, \beta, \theta, \gamma)=1-\left(\left[1-e^{-\theta x^{\gamma}}\right]^{\alpha}\left[\beta+(1-\beta)\left(1-e^{-\theta x^{\gamma}}\right)\right]^{-\alpha}\right),
$$

and

$$
h(x ; \alpha, \beta, \theta, \gamma)=\frac{\left[\alpha \beta \theta \gamma x^{\gamma-1} e^{-\theta x^{\gamma}}\left(1-e^{-\theta x^{\gamma}}\right)^{\alpha-1}\left[\beta+(1-\beta)\left(1-e^{-\theta x^{\gamma}}\right)\right]^{\alpha-1}\right]}{1-\left(\left[1-e^{-\theta x^{\gamma}}\right]^{\alpha}\left[\beta+(1-\beta)\left(1-e^{-\theta x^{\gamma}}\right)\right]^{-\alpha}\right)} .
$$

Figure 1, 2 and 3 illustrate some possible shapes of the pdf, cdf and hazard function of OIP-W distribution for some values of the parameters $\alpha, \beta, \theta$ and $\gamma$.

Note that the OIP-W distribution is very flexible model that approaches to different distributions when its parameters are changed. The OIP-W distribution contains following well known 


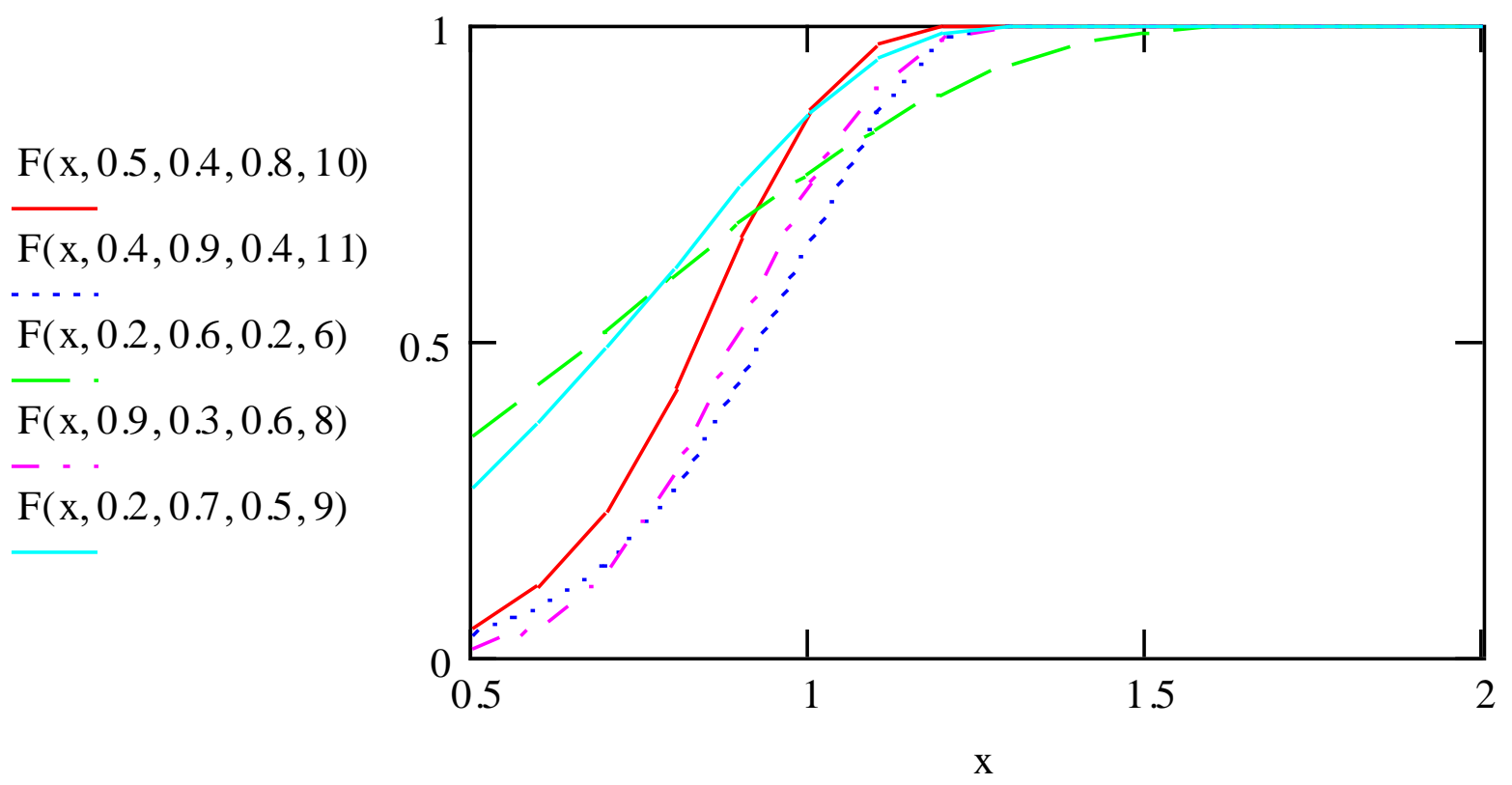

Figure 2: The cdfs of OIP-W distribution with different parameters.

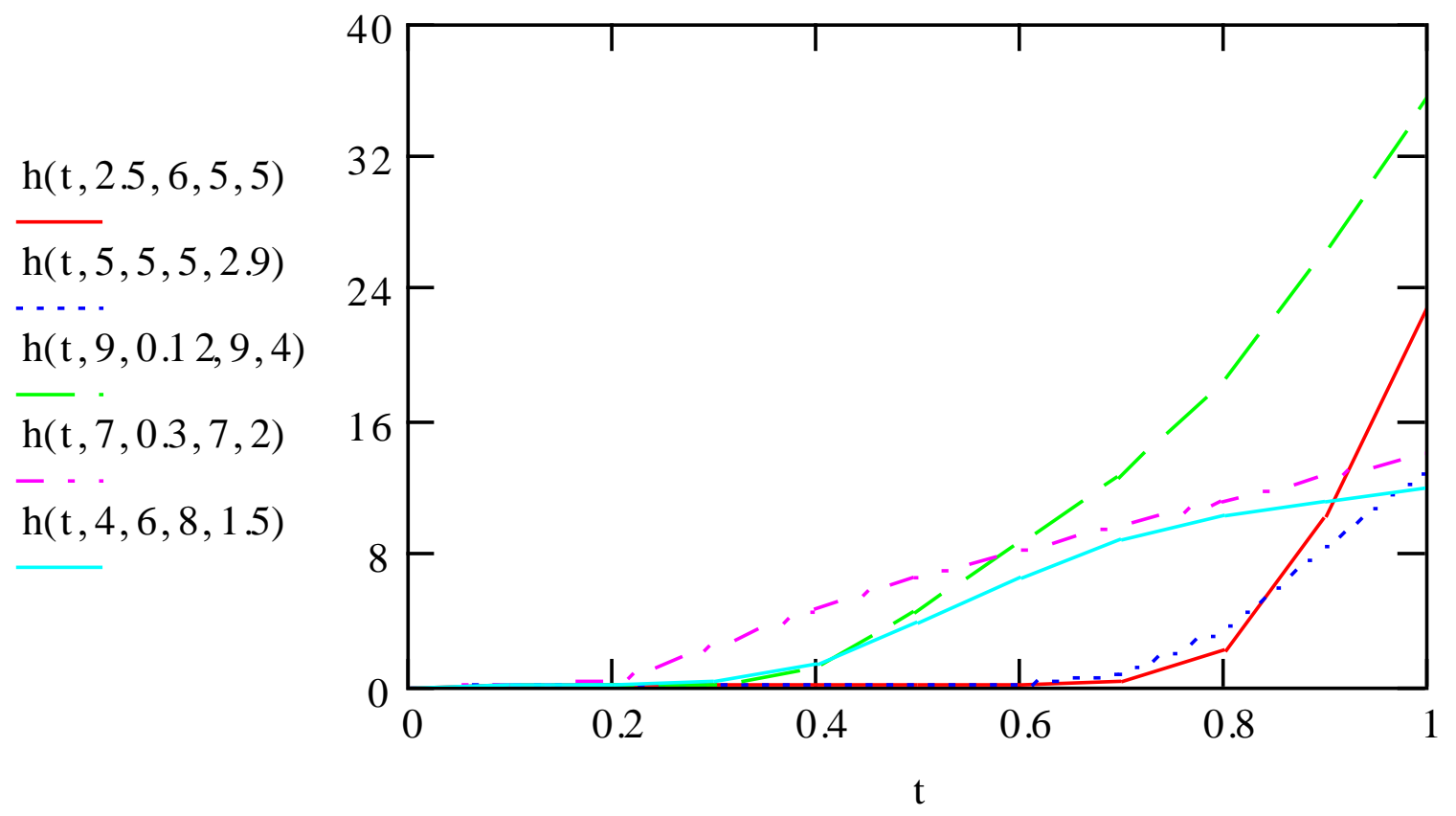

Figure 3: The hazard functions of OIP-W distribution with different parameters. 
distributions as its special cases. In particular, when $\beta=1$ the OIP-W distribution reduces to the exponentiated-weibull (EXP-W) distribution (Mudholkar and Srivastava, 1993). When $\alpha=1$, The OIP-W distribution reduces to Marshall-Olkin-Weibull (MO-W) distribution. When $\beta=\gamma=1$, then the resulting distribution is the exponentiated-exponential distribution. When $\alpha=\gamma=1$, we get the Marshall-Olkin-exponential distribution. Clearly, when $\alpha=\beta=\gamma=1$, we obtain the weibull distribution. Finally, when $\alpha=\beta=\gamma=1$, then the resulting distribution is the exponential distribution.

\section{Statistical Properties}

In this section, some statistical properties for the OIP-W distribution are obtained.

\subsection{Useful Expansions}

In this subsection, some useful expansions of pdf and cdf for OIP-W distribution are covered.

Firstly, we obtain an expansion for pdf of OIP-W distribution as a linear combination of weibull distribution. Using the power series

$$
(1+d z)^{-t}=\sum_{k=0}^{\infty}\left(\begin{array}{c}
-t \\
k
\end{array}\right) d^{t} z^{t}
$$

for the last term and inserting the resulting expansion in (4) gives:

$$
f(x ; \alpha, \beta, \theta, \gamma)=\alpha \theta \gamma x^{\gamma-1} e^{-\theta x^{\gamma}} \sum_{k=0}^{\infty}\left(\begin{array}{c}
-\alpha-1 \\
k
\end{array}\right) \beta^{-\alpha-k}(1-\beta)^{k}\left(1-e^{-\theta x^{\gamma}}\right)^{\alpha+k-1} .
$$

For $|z|<1$, the power series holds

$$
(1-z)^{-t}=\sum_{j=0}^{\infty}(-1)^{j}\left(\begin{array}{c}
-t \\
j
\end{array}\right) z^{t}
$$

Applying (6) to last term of (5), gives

$$
f(x ; \alpha, \beta, \theta, \gamma)=\sum_{k, j=0}^{\infty} \psi_{k, j} \theta \gamma x^{\gamma-1} e^{-\theta(j+1) x^{\gamma}},
$$

where

$$
\psi_{k, j}=\alpha \sum_{k, j=0}^{\infty}(-1)^{j}\left(\begin{array}{c}
-\alpha-1 \\
k
\end{array}\right)\left(\begin{array}{c}
\alpha+k-1 \\
j
\end{array}\right) \beta^{-\alpha-k}(1-\beta)^{k} .
$$

Secondly, an expansion of $[F(x)]^{h}$ is obtained as following, Now

$$
[F(x)]^{h}=\left[1-e^{-\theta x^{\gamma}}\right]^{\alpha h}\left[\beta+(1-\beta)\left(1-e^{-\theta x^{\gamma}}\right)\right]^{-\alpha h},
$$

We can rewrite the last term as

$$
\left[\beta+(1-\beta)\left(1-e^{-\theta x^{\gamma}}\right)\right]^{-\alpha h}=\sum_{t=0}^{\infty}\left(\begin{array}{c}
-\alpha h \\
t
\end{array}\right) \beta^{-\alpha h-t}(1-\beta)^{t}\left(1-e^{-\theta x^{\gamma}}\right)^{t} .
$$


Then

$$
[F(x)]^{h}=\sum_{t=0}^{\infty}\left(\begin{array}{c}
-\alpha h \\
t
\end{array}\right) \beta^{-\alpha h-t}(1-\beta)^{t}\left(1-e^{-\theta x^{\gamma}}\right)^{\alpha h+t} .
$$

Using binomial expansion, we get

$$
[F(x)]^{h}=\sum_{t, l=0}^{\infty} \eta_{t, l} e^{-\theta l x^{\gamma}}
$$

where

$$
\eta_{t, l}=\sum_{t, l=0}^{\infty}(-1)^{l}\left(\begin{array}{c}
-\alpha h \\
t
\end{array}\right)\left(\begin{array}{c}
\alpha h+t \\
l
\end{array}\right) \beta^{-\alpha h-t}(1-\beta)^{t} .
$$

\subsection{Quantile and Median}

The quantile function $x_{q}$ of OIP-W distribution is

$$
x_{q}=\left[\frac{-1}{\theta} \ln \left(1-\left[\frac{\beta q^{\frac{1}{\alpha}}}{1-(1-\beta) q^{\frac{1}{\alpha}}}\right]\right)\right]^{\frac{1}{\gamma}}, \quad 0 \leq q \leq 1 .
$$

By putting $q=0.5$ in formula (9) we can get the median of odd inverse Pareto-Weibull distribution.

\subsection{Ordinary Moments}

If $\mathrm{X}$ has the pdf (7), the $r$ th moment of the OIP-W distribution can be calculated through the following relation.

$$
\mu_{r}^{\prime}=E\left(X^{r}\right)=\int_{0}^{\infty} x^{r} f(x) d x
$$

Substituting (7) into (10), we obtain

$$
\mu_{r}^{\prime}=\sum_{k, j=0}^{\infty} \psi_{k, j} \theta \gamma \int_{0}^{\infty} x^{r+\gamma-1} e^{-\theta(j+1) x^{\gamma}} d x
$$

Let $y=\theta(j+1) x^{\gamma}$, then the moments becomes

$$
\mu_{r}^{\prime}=\sum_{k, j=0}^{\infty} \frac{\psi_{k, j}}{\theta^{\frac{r}{\gamma}}(j+1)^{\frac{r}{\gamma}+1}} \Gamma\left(\frac{r}{\gamma}+1\right)
$$

The moment generating function of the OIP-W distribution is obtained as

$$
M_{X}(t)=\sum_{r=0}^{\infty} \frac{t^{r}}{r !} E\left(X^{r}\right)=\sum_{k, j, r=0}^{\infty} \frac{t^{r}}{r !} \frac{\psi_{k, j}}{\theta^{\frac{r}{\gamma}}(j+1)^{\frac{r}{\gamma}+1}} \Gamma\left(\frac{r}{\gamma}+1\right) .
$$




\subsection{Incomplete and Conditional Moments}

If X has the pdf (7), the sth incomplete moment of the OIP-W distribution can be calculated as follows:

$$
m_{s}(z)=\int_{0}^{z} x^{s} f(x) d x=\sum_{k, j=0}^{\infty} \psi_{k, j} \theta \gamma \int_{0}^{z} x^{s+\gamma-1} e^{-\theta(j+1) x^{\gamma}} d x
$$

Using lower incomplete gamma function, we get

$$
m_{s}(z)=\sum_{k, j=0}^{\infty} \frac{\psi_{k, j}}{\theta^{\frac{s}{\gamma}}(j+1)^{\frac{s}{\gamma}+1}} v\left(\frac{s}{\gamma}+1, \theta(j+1) z^{\gamma}\right),
$$

where $v(s, z)=\int_{0}^{z} x^{s-1} e^{-x} d x$ is the lower incomplete gamma function. Further, the conditional moments of the OIP-W distribution can be calculated from the following relation:

$$
\Delta_{s}(z)=\int_{z}^{\infty} x^{s} f(x) d x=\sum_{k, j=0}^{\infty} \psi_{k, j} \theta \gamma \int_{z}^{\infty} x^{s+\gamma-1} e^{-\theta(j+1) x^{\gamma}} d x
$$

Using upper incomplete gamma function, we get

$$
\Delta_{s}(z)=\sum_{k, j=0}^{\infty} \frac{\psi_{k, j}}{\theta^{\frac{s}{\gamma}}(j+1)^{\frac{s}{\gamma}+1}} \Gamma\left(\frac{s}{\gamma}+1, \theta(j+1) z^{\gamma}\right),
$$

where $\Gamma(s, z)=\int_{z}^{\infty} x^{s-1} e^{-x} d x$, is the upper incomplete gamma function.

\subsection{Probability Weighted Moment}

The probability weighted moment (PWM) can be obtained using the following relation:

$$
\tau_{r, s}=E\left[X^{r} F(x)^{s}\right]=\int_{-\infty}^{\infty} x^{r} f(x)(F(x))^{s} d x .
$$

The PWM of the OIP-W distribution is obtained by substituting (7) and (8) into (11), replacing $h$ with $s$, leads to

$$
\begin{aligned}
\tau_{r, s} & =\int_{0}^{\infty} \sum_{k, j, t, l=0}^{\infty} \psi_{k, j} \eta_{t, l} \theta \gamma x^{r+\gamma-1} e^{-\theta(j+l+1) x^{\gamma}} d x \\
& =\sum_{k, j, t, l=0}^{\infty} \psi_{k, j} \eta_{t, l} \frac{1}{\theta^{r / \gamma}(j+l+1)^{(r / \gamma)+1}} \Gamma\left(\frac{r}{\gamma}+1\right) .
\end{aligned}
$$

\subsection{Inequality Measures}

The first incomplete moment can be used to construct inequality measures called Lorenz and Bonferroni curves, which are most widely used in income and wealth distributions. The Lorenz and Bonferroni curves of the OIP-W distribution are obtained as follows:

$$
L_{F}(x)=\frac{\int_{0}^{z} x f(x) d x}{E(X)}=\frac{\sum_{k, j=0}^{\infty} \frac{\psi_{k, j}}{\theta^{1 / \gamma}(j+1)^{(1 / \gamma)+1}} v\left(\frac{1}{\gamma}+1, \theta(j+1) z^{\gamma}\right)}{\sum_{k, j=0}^{\infty} \frac{\psi_{k, j}}{\theta^{1 / \gamma}(j+1)^{(1 / \gamma)+1}} \Gamma\left(\frac{1}{\gamma}+1\right)},
$$


and

$$
B_{F}(x)=\frac{\int_{0}^{z} x f(x) d x}{E(X) F(x)}=\frac{\sum_{k, j=0}^{\infty} \frac{\psi_{k, j}}{\theta^{1 / \gamma}(j+1)^{(1 / \gamma)+1}} v\left(\frac{1}{\gamma}+1, \theta(j+1) z^{\gamma}\right)}{\sum_{k, j=0}^{\infty} \frac{\psi_{k, j}}{\theta^{1 / \gamma}(j+1)^{(1 / \gamma)+1}} \Gamma\left(\frac{1}{\gamma}+1\right)\left\{\frac{\left(1-e^{-\theta x^{\gamma}}\right)}{\beta+(1-\beta)\left(1-e^{-\theta x^{\gamma}}\right)}\right\}^{\alpha}} .
$$

\subsection{Rènyi and q-Entropies}

The Rènyi entropy of a random variable (r.v.) $X$ represents a measure of variation of the uncertainty. The Rènyi entropy is defined by:

$$
I_{R}(X)=\frac{1}{1-\lambda} \log \left\{\int_{-\infty}^{\infty} f^{\lambda}(x) d x\right\}, \quad \lambda>0, \quad \lambda \neq 1 .
$$

Therefore, the Rènyi entropy of a r.v. $X$ which follows the OIP-W is given by:

$$
I_{R}(X)=\frac{1}{1-\lambda} \log \left[\frac{\gamma^{\lambda-1} \theta^{\frac{\lambda}{\gamma}+\gamma-2}}{(\lambda(j+1))^{\frac{\lambda}{\gamma}(\gamma-1)-\gamma+2}} \sum_{k, j=0}^{\infty} \psi_{k, j} \Gamma\left(\frac{\lambda}{\gamma}(\gamma-1)-\gamma+2\right)\right] .
$$

The q-entropy say, $H_{q}(X)$, is defined by:

$$
H_{q}(X)=\frac{1}{q-1} \log \left\{1-\int_{-\infty}^{\infty} f^{q}(x) d x\right\}, \quad q>0, \quad q \neq 1 .
$$

For the OIP-W distribution, the q-entropy is defined as follows:

$$
H_{q}(X)=\frac{1}{q-1} \log \left\{1-\left[\frac{\gamma^{q-1} \theta^{\frac{q}{\gamma}+\gamma-2}}{(q(j+1))^{\frac{q}{\gamma}(\gamma-1)-\gamma+2}} \sum_{k, j=0}^{\infty} \psi_{k, j} \Gamma\left(\frac{q}{\gamma}(\gamma-1)-\gamma+2\right)\right]\right\}
$$

\section{Order Statistic}

Let $X_{1}, X_{2}, \ldots, X_{n}$ be a simple random sample of size $\mathrm{n}$ from OIP-W distribution and $X_{(1)}$, $X_{(2)}, \ldots, X_{(n)}$ be the corresponding order statistics. The pdf of $X_{(r)}$ is given by

$$
f_{(r)}(x)=\frac{1}{B(r, n-r+1)} f(x) \sum_{i=0}^{n-r}\left(\begin{array}{c}
n-r \\
i
\end{array}\right)(-1)^{i}[F(x)]^{i+r-1} .
$$

where $F(x)$ and $f(x)$ are the cdf and pdf of the OIP-W distribution given by (3) and (4) respectively. $B(.,$.$) is the beta function. Based on equations (7) and (8), we can write$

$$
\begin{aligned}
f(x) F(x)^{i+r-1}= & \theta \gamma \sum_{k, j, t, l=0}^{\infty} \frac{(-1)^{l}(1-\beta)^{t}}{\beta^{\alpha(i+r-1)+t}}\left(\begin{array}{c}
-\alpha(i+r-1) \\
t
\end{array}\right)\left(\begin{array}{c}
\alpha(i+r-1)+t \\
l
\end{array}\right) \\
& \times \psi_{k, j} x^{\gamma-1} e^{-\theta(j+l+1) x^{\gamma}} .
\end{aligned}
$$

Inserting (13) into (12), the pdf of $X_{(r)}$ could be written as

$$
f_{(r)}(x)=\sum_{k, j, t, l=0}^{\infty} \omega_{k, j, t, l} x^{\gamma-1} e^{-\theta(j+l+1) x^{\gamma}},
$$

where

$$
\omega_{k, j, t, l}=\sum_{i=0}^{n-r} \frac{(-1)^{i+l} \theta \gamma(1-\beta)^{t}}{\beta^{\alpha(i+r-1)+t} B(r, n-r+1)}\left(\begin{array}{c}
n-r \\
i
\end{array}\right)\left(\begin{array}{c}
-\alpha(i+r-1) \\
t
\end{array}\right)\left(\begin{array}{c}
\alpha(i+r-1)+t \\
l
\end{array}\right) \psi_{k, j} .
$$




\section{Maximum Likelihood Estimation}

In this section, we discuss the estimation of the unknown parameters of the OIP-W distribution from complete sample by using the method of maximum likelihood. Let $X_{1}, X_{2}, \ldots, X_{n}$ be a random sample of size $\mathrm{n}$ from $\mathrm{OIP}-\mathrm{W}(\zeta)$, where $\zeta=(\alpha, \beta, \theta, \gamma)$. The log-likelihood function $\mathrm{L}$ is given by:

$$
\begin{aligned}
L= & n \ln \alpha+n \ln \beta+n \ln \theta+n \ln \gamma+(\gamma-1) \sum_{i=1}^{n} \ln \left(x_{i}\right)-\theta \sum_{i=1}^{n}\left(x_{i}^{\gamma}\right) \\
& +(\alpha-1) \sum_{i=1}^{n} \ln \left(1-e^{-\theta x_{i}^{\gamma}}\right)-(\alpha+1) \sum_{i=1}^{n} \ln \left[\beta+(1-\beta) e^{-\theta x_{i}^{\gamma}}\right] .
\end{aligned}
$$

The maximum likelihood estimates (MLEs) of the parameters are obtained by Differentiating the log-likelihood function $\mathrm{L}$ with respect to the parameters $\alpha, \beta, \theta$ and $\gamma$ and setting the results to zero.

$$
\begin{gathered}
\frac{\partial L}{\partial \alpha}=\frac{n}{\alpha}+\sum_{i=1}^{n} \ln \phi\left(x_{i}, \theta, \gamma\right)-\sum_{i=1}^{n} \ln \left[\beta+(1-\beta) \phi\left(x_{i}, \theta, \gamma\right)\right]=0 \\
\frac{\partial L}{\partial \beta}=\frac{n}{\beta}-(\alpha+1) \sum_{i=1}^{n}\left[\frac{e^{-\theta x_{i}^{\gamma}}}{\left[\beta+(1-\beta) \phi\left(x_{i}, \theta, \gamma\right)\right]}\right]=0 \\
\frac{\partial L}{\partial \theta}=\frac{n}{\theta}-\sum_{i=1}^{n} x_{i}^{\gamma}+(\alpha-1) \sum_{i=1}^{n}\left[\frac{x_{i}^{\gamma} e^{-\theta x_{i}^{\gamma}}}{\phi\left(x_{i}, \theta, \gamma\right)}\right]-(\alpha+1) \sum_{i=1}^{n}\left[\frac{(1-\beta) x_{i}^{\gamma} e^{-\theta x_{i}^{\gamma}}}{\left[\beta+(1-\beta) \phi\left(x_{i}, \theta, \gamma\right)\right]}\right]=0 \\
\frac{\partial L}{\partial \gamma}=\frac{n}{\gamma}+\sum_{i=1}^{n} \ln \left(x_{i}\right)-\theta \sum_{i=1}^{n}\left(x_{i}^{\gamma} \ln \left(x_{i}\right)\right)+(\alpha-1) \sum_{i=1}^{n}\left[\frac{\theta x_{i}^{\gamma} \ln \left(x_{i}\right) e^{-\theta x_{i}^{\gamma}}}{\phi\left(x_{i}, \theta, \gamma\right)}\right] \\
-(\alpha+1) \times \sum_{i=1}^{n}\left[\frac{\left.(1-\beta) \theta x_{i}^{\gamma} \ln \left(x_{i}\right) e^{-\theta x_{i}^{\gamma}}\right]=0}{\left[\beta+(1-\beta) \phi\left(x_{i}, \theta, \gamma\right)\right]}\right]
\end{gathered}
$$

where $\phi\left(x_{i}, \theta, \gamma\right)=\left(1-e^{-\theta x_{i}^{\gamma}}\right)$. From (14), we obtain the MLE of $\alpha$ in a closed form as

$$
\hat{\alpha}=\frac{n}{\left[\sum_{i=1}^{n} \ln \left[\hat{\beta}+(1-\hat{\beta}) \phi\left(x_{i}, \hat{\theta}, \hat{\gamma}\right)\right]-\sum_{i=1}^{n} \ln \left(\phi\left(x_{i}, \hat{\theta}, \hat{\gamma}\right)\right)\right]} .
$$

Substituting from (18) into (15), (16), and (17), we get the MLEs of $(\beta, \theta, \gamma)$ by solving the resulting system of non-linear equations. These equations cannot be solved analytically. We apply statistical software such as Mathcad to solve them numerically.

The elements of the observed information matrix are:

$$
\begin{aligned}
\frac{\partial^{2} L}{\partial \alpha^{2}} & =\frac{-n}{\alpha^{2}}, \\
\frac{\partial^{2} L}{\partial \alpha \partial \beta} & =-\sum_{i=1}^{n}\left[\frac{e^{-\theta x_{i}^{\gamma}}}{\left[\beta+(1-\beta) \phi\left(x_{i}, \theta, \gamma\right)\right]}\right], \\
\frac{\partial^{2} L}{\partial \alpha \partial \theta} & =\sum_{i=1}^{\infty}\left[\frac{x_{i}^{\gamma} e^{-\theta x_{i}^{\gamma}}}{\phi\left(x_{i}, \theta, \gamma\right)}\right]-\sum_{i=1}^{n}\left[\frac{(1-\beta) x_{i}^{\gamma} e^{-\theta x_{i}^{\gamma}}}{\left[\beta+(1-\beta) \phi\left(x_{i}, \theta, \gamma\right)\right]}\right],
\end{aligned}
$$




$$
\begin{aligned}
& \frac{\partial^{2} L}{\partial \beta^{2}}=\frac{-n}{\beta^{2}}+(\alpha+1) \sum_{i=1}^{n}\left[\frac{e^{-\theta x_{i}^{\gamma}}}{\left[\beta+(1-\beta) \phi\left(x_{i}, \theta, \gamma\right)\right]}\right]^{2}, \\
& \frac{\partial^{2} L}{\partial \beta \partial \theta}=(\alpha+1) \sum_{i=1}^{n}\left[\frac{x_{i}^{\gamma} e^{-\theta x_{i}^{\gamma}}}{\left[\beta+(1-\beta) \phi\left(x_{i}, \theta, \gamma\right)\right]}\right]\left[1+\frac{(1-\beta) e^{-\theta x_{i}^{\gamma}}}{\left[\beta+(1-\beta) \phi\left(x_{i}, \theta, \gamma\right)\right]}\right] \text {, } \\
& \frac{\partial^{2} L}{\partial \beta \partial \gamma}=(\alpha+1) \sum_{i=1}^{n}\left[\frac{\theta x_{i}^{\gamma} \ln \left(x_{i}\right) e^{-\theta x_{i}^{\gamma}}}{\left[\beta+(1-\beta) \phi\left(x_{i}, \theta, \gamma\right)\right]}\right]\left[1-\frac{(1-\beta) e^{-\theta x_{i}^{\gamma}}}{\left[\beta+(1-\beta) \phi\left(x_{i}, \theta, \gamma\right)\right]}\right] \text {, } \\
& \frac{\partial^{2} L}{\partial \theta^{2}}=\frac{-n}{\theta^{2}}-(\alpha-1) \sum_{i=1}^{n}\left[\frac{x_{i}^{2 \gamma} e^{-\theta x_{i}^{\gamma}}}{\phi\left(x_{i}, \theta, \gamma\right)}\left[1+\frac{e^{-\theta x_{i}^{\gamma}}}{\phi\left(x_{i}, \theta, \gamma\right)}\right]\right]+ \\
& (\alpha+1) \sum_{i=1}^{n}\left[\frac{(1-\beta) x_{i}^{\gamma} e^{-\theta x_{i}^{\gamma}}}{\left[\beta+(1-\beta) \phi\left(x_{i}, \theta, \gamma\right)\right]}\right]\left[x_{i}^{\gamma}+\frac{(1-\beta) x_{i}^{\gamma} e^{-\theta x_{i}^{\gamma}}}{\left[\beta+(1-\beta) \phi\left(x_{i}, \theta, \gamma\right)\right]}\right] \text {, } \\
& \frac{\partial^{2} L}{\partial \theta \partial \gamma}=(\alpha-1) \sum_{i=1}^{n}\left[\frac{x_{i}^{\gamma} \ln \left(x_{i}^{\gamma}\right) e^{-\theta x_{i}^{\gamma}}}{\phi\left(x_{i}, \theta, \gamma\right)}\left[1-\theta x_{i}^{\gamma}-\frac{\theta x_{i}^{\gamma} e^{-\theta x_{i}^{\gamma}}}{\phi\left(x_{i}, \theta, \gamma\right)}\right]\right]-\sum_{i=1}^{n} x_{i}^{\gamma} \ln \left(x_{i}^{\gamma}\right)- \\
& (\alpha+1) \sum_{i=1}^{n}\left[\frac{(1-\beta) x_{i}^{\gamma} \ln \left(x_{i}^{\gamma}\right) e^{-\theta x_{i}^{\gamma}}}{\left[\beta+(1-\beta) \phi\left(x_{i}, \theta, \gamma\right)\right]}\right]\left[1-\theta x_{i}^{\gamma}-\frac{(1-\beta) \theta x_{i}^{\gamma} e^{-\theta x_{i}^{\gamma}}}{\left[\beta+(1-\beta) \phi\left(x_{i}, \theta, \gamma\right)\right]}\right] \text {, } \\
& \frac{\partial^{2} L}{\partial \gamma^{2}}=\frac{-n}{\gamma^{2}}-\theta \sum_{i=1}^{n} x_{i}^{\gamma}\left[\ln \left(x_{i}\right)\right]^{2}+(\alpha-1) \sum_{i=1}^{n}\left[\frac{\theta x_{i}^{\gamma}\left[\ln \left(x_{i}\right)\right]^{2} e^{-\theta x_{i}^{\gamma}}}{\phi\left(x_{i}, \theta, \gamma\right)}\left[1-\theta x_{i}^{\gamma}-\frac{\theta x_{i}^{\gamma} e^{-\theta x_{i}^{\gamma}}}{\phi\left(x_{i}, \theta, \gamma\right)}\right]\right]- \\
& (\alpha+1) \sum_{i=1}^{n}\left[\frac{(1-\beta) \theta x_{i}^{\gamma}\left[\ln \left(x_{i}\right)\right]^{2} e^{-\theta x_{i}^{\gamma}}}{\left[\beta+(1-\beta) \phi\left(x_{i}, \theta, \gamma\right)\right]}\right]\left[1-\theta x_{i}^{\gamma}-\frac{(1-\beta) \theta x_{i}^{\gamma} e^{-\theta x_{i}^{\gamma}}}{\left[\beta+(1-\beta) \phi\left(x_{i}, \theta, \gamma\right)\right]}\right] \text {. }
\end{aligned}
$$

Approximate two-sided 100(1-v)\% confidence intervals of the unknown parameters $(\alpha, \beta, \theta, \gamma)$ are $\quad \hat{\alpha} \pm z_{\frac{v}{2}} \sqrt{\operatorname{Var}(\hat{\alpha})}, \quad \hat{\beta} \pm z_{\frac{\nu}{2}} \sqrt{\operatorname{Var}(\hat{\beta})}, \quad \hat{\theta} \pm z_{\frac{v \delta}{2}} \sqrt{\operatorname{Var}(\hat{\theta})}, \quad$ and $\hat{\gamma} \pm z_{\frac{v}{2}} \sqrt{\operatorname{Var}(\hat{\gamma})}$, respectively where $z_{\frac{v}{2}}$ is the upper $\left(\frac{v}{2}\right)$ th percentile of the standard normal distribution.

\section{Applications}

In this section, we perform an application to a real data set to evaluate the flexibility of the OIP$\mathrm{W}$ model. In order to compare the OIP-W distribution with other fitted distributions, including Odd log-logistic Weibull (OLLW), Kumaraswamy Weibull (KwW), Beta-Weibull (BW), Odd log-logistic Marshall Olkin Weibull (OLLMOW), and McDonald Weibull (McW). The data set is taken from Van Montfort (1970) which represents the Maximum Annual Flood Discharges of the North Saskachevan in units of 1000 cubic feet per second, of the North Saskachevan River at Edmonton, over a period of 48 years. The data are in Table 1. The MLEs that are computed using adequacy model is given in Table 2. The goodness of fit measures including the values of log-likelihood function, Akaike Information Criteria (AIC), Bayesian Information Criteria (BIC), and Corrected Akaike Information Criteria (CAIC) are given in Table 3 for the five models in order to verify which distribution fits better to these the data. Generally, we consider the best fit using the smaller values of these statistics. Table 3 show that the OIP-W model has the lowest values of all statistical measures among all fitted distributions. 
Table 1: The data.

\begin{tabular}{rrrrrrrrr}
\hline 19.885 & 20.940 & 21.820 & 23.700 & 24.888 & 25.460 & 25.760 & 26.720 & 27.500 \\
28.100 & 28.600 & 30.200 & 30.380 & 31.500 & 32.600 & 32.680 & 34.400 & 35.347 \\
35.700 & 38.100 & 39.020 & 39.200 & 40.000 & 40.400 & 40.400 & 42.250 & 44.020 \\
44.730 & 44.900 & 46.300 & 50.330 & 51.442 & 57.220 & 58.700 & 58.800 & 61.200 \\
61.740 & 65.440 & 65.597 & 66.000 & 74.100 & 75.800 & 84.100 & 106.600 & 109.700 \\
121.970 & 121.970 & 185.560 & & & & & & \\
\hline
\end{tabular}

Table 2: MLE values from fitting the data set.

\begin{tabular}{cccccc}
\hline Model & \multicolumn{5}{c}{ Estimates } \\
\hline OLLW & 0.107 & 0.494 & 4.878 & - & - \\
KwW & 0.049 & 1.175 & 8.908 & 0.306 & - \\
BW & 0.074 & 1.061 & 8.887 & 0.373 & - \\
OLLMOW & 0.138 & 0.423 & 5.580 & 0.874 & - \\
McW & 0.134 & 1.041 & 5.404 & 0.216 & 4.820 \\
OIPW & 1.497 & 7.410 & 1.617 & 0.371 & - \\
\hline
\end{tabular}

Table 3: Goodness of fit measures for the data set.

\begin{tabular}{ccccc}
\hline Model & \multicolumn{4}{c}{ Measures } \\
\cline { 2 - 5 } & $-2 \mathrm{~L}$ & AIC & BIC & CAIC \\
\hline OLLW & -438.300 & 444.300 & 449.914 & 443.846 \\
KwW & -435.443 & 443.443 & 450.928 & 444.373 \\
BW & -435.190 & 443.190 & 450.673 & 444.121 \\
OLLMOW & -438.060 & 446.060 & 453.544 & 446.989 \\
McW & -432.272 & 442.372 & 451.728 & 443.800 \\
OIPW & -431.607 & 439.607 & 447.151 & 440.596 \\
\hline
\end{tabular}




\section{Conclusion}

In this paper, a new four-parameter model, which is called OIP-W distribution, is introduced. Explicit expressions for some statistical properties of the distribution are derived and discussed. Maximum likelihood estimation is derived and the observed fisher information matrix is obtained. The OIP-W distribution is employed to fit a real data set, and it performs better than some other competitive distributions.

\section{References}

Aldahlan MA, Afify AZ, Ahmed AHN (2019). The odd inverse Pareto-G class: Properties and applications. Journal of Nonlinear Sciences 65 Applications, 12(5): 278-290.

Alzaatreh A, Lee C, Famoye F (2013). A new method for generating families of continuous distributions. Metron, 71(1): 63-79.

Bourguignon M, Silva RB, Cordeiro GM (2014). The Weibull-G family of probability distributions. Journal of Data Science, 12(1): 53-68.

Cordeiro GM, Afify AZ, Ortega EM, Suzuki AK, Mead ME (2019). The odd Lomax generator of distributions: Properties, estimation and applications. Journal of Computational and Applied Mathematics, 347: 222-237.

Cordeiro GM, Afify AZ, Yousof HM, Pescim RR, Aryal GR (2017). The exponentiated Weibull$\mathrm{H}$ family of distributions: Theory and applications. Mediterranean Journal of Mathematics, 14(4): 1-22.

Cordeiro GM, Ortega EM, Popović BV, Pescim RR (2014). The Lomax generator of distributions: Properties, minification process and regression model. Applied Mathematics and Computation, 247: 465-486.

Eugene N, Lee C, Famoye F (2002). Beta-normal distribution and its applications. Communications in Statistics: Theory and methods, 31(4): 497-512.

Gupta RC, Gupta PL, Gupta RD (1998). Modeling failure time data by Lehman alternatives. Communications in Statistics: Theory and methods, 27(4): 887-904.

Marshall AW, Olkin I (1997). A new method for adding a parameter to a family of distributions with application to the exponential and Weibull families. Biometrika, 84(3): 641-652.

Mudholkar GS, Srivastava DK (1993). Exponentiated Weibull family for analyzing bathtub failure-rate data. Institute of Electrical and Electronics Engineers transactions on reliability, 42(2): 299-302.

Torabi H, Hedesh NM (2012). The gamma-uniform distribution and its applications. Kybernetika, 48(1): 16-30.

Van Montfort M (1970). On testing that the distribution of extremes is of type I when type II is the alternative. Journal of Hydrology, 11(4): 421-427.

Zografos K, Balakrishnan N (2009). On families of beta-and generalized gamma-generated distributions and associated inference. Statistical Methodology, 6(4): 344-362. 